\title{
Chemical Studies of Partially Hydrolysed Lipopolysaccharides from Four Strains of Campylobacter jejuni and Two Strains of Campylobacter coli
}

\author{
By VIGFRID N $Æ S S$ AND TOR HOFSTAD* \\ Department of Microbiology and Immunology. The Gade Institute, University of Bergen. \\ Bergen. Norway
}

(Received 20 March 1984; revised 29 May 1984)

Lipopolysaccharides (LPS) from four strains of Campylobacter jejuni and two strains of $C$. coli were partially hydrolysed with $1 \%$ acetic acid. Subsequent chloroform extraction led to the formation of a polysaccharide-containing aqueous layer, an interfacial material and a lipid Acontaining chloroform layer. The polysaccharides contained the neutral sugars, amino sugars, 2-keto-3-deoxy-octonic acid, and part of the phosphorus present in the undegraded LPS. The lipid As were made up of glucosamine, phosphorus, ester- and,amide-linked 3-hydroxytetradecanoic acid, and ester-linked $n$-tetradecanoic and $n$-hexadecanoic acid. The interfacial material was made up of lipid $A$ and undegraded LPS.

When chromatographed on Bio-Gel P-60, the degraded polysaccharides were eluted as two incompletely separated peaks (strains NCTC 11168, NCTC 11351, 11041 and 11101) or as one peak (strains NCTC 11392 and E 8035). All peaks appeared close to the total volume of the column. When the different fractions were re-chromatographed on Bio-Gel P-10, the peaks still appeared close to the total volume of the column. These findings indicate that LPS from $C$. jejuni and $C$. coli are devoid of long $\mathrm{O}$-antigenic side-chains.

\section{INTRODUCTION}

Lipopolysaccharides (LPS) have been isolated from eight strains of Campylobacter jejuni and three strains of $C$. coli (Næss \& Hofstad, 1982, 1984). The chemical composition resembled that of cell-wall LPS isolated from other Gram-negative, enteric bacteria. Phosphorus, ethanolamine, 3-hydroxytetradecanoic acid, n-tetradecanoic acid, n-hexadecanoic acid, 2-keto-3-deoxyoctonate, L-glycero-D-manno-heptose, glucose, galactose and glucosamine were present in all Campylobacter LPS. They varied with respect to the presence or absence of mannose and galactosamine. The number of neutral sugar residues relative to fatty acid residues was low (less than 2.5), and the molar ratios of galactose and glucose relative to L-glycero-D-manno-heptose varied from $0 \cdot 5-3.0$ to $1 \cdot 0$ and $0 \cdot 6-4 \cdot 1$ to $1 \cdot 0$, respectively. These findings indicate that if the LPS core oligosaccharide is substituted by antigenic side-chain units, the number of units present must be low.

Samples of the isolated LPS were degraded by treatment with $1 \%$ acetic acid to obtain information on the distribution of the components present. The isolated polysaccharide-containing aqueous layer was fractionated by gel filtration on Bio-Gel columns. The present paper deals with the degradation and subsequent gel filtration experiments carried out with LPS isolated from four strains of $C$. jejuni and two strains of $C$. coli, and with the chemical composition of the various materials thus obtained.

Abbreviations: KDO, 2-keto-3-deoxy-octonate; TFAA, trifluoroacetic anhydride in acetonitrile. 


\section{METHODS}

Bacterial strains and cultication. Campylobacter jejuni strains 11041, NCTC 11168, NCTC 11392 and NCTC II351 (CIP 702) and C. coli strains 11101 and E 8035 were cultivated on plates of Tryptose agar (Difco) with the FBP supplement described by George et al. (1978), menadione (10 $\mu \mathrm{g} \mathrm{ml} \mathrm{m}^{-1}$ ) and $7 \%$ (v/v) human blood. The plates were incubated in a $2.51 \mathrm{BBL}$ GasPak jar with one $\mathrm{GasPak} \mathrm{H}_{2}-\mathrm{CO}_{2}$ envelope and without a catalyst at $37^{\circ} \mathrm{C}$ for 3-4 d. The bacteria were harvested by washing the growth off the agar using a right-angled glass rod and $1 \%(v / v)$ formaldehyde in sterile saline. Following centrifugation (10000 for $20 \mathrm{~min})$ the organisms were washed in sterile saline before being stored as a paste at $-20^{\circ} \mathrm{C}$ until used.

Chemicals. The solvents used were of p.a. grade (Merck). Saturated methanolic $\mathrm{HCl}$ was produced from concentrated $\mathrm{HCl}$, concentrated $\mathrm{H}_{2} \mathrm{SO}_{4}$ and methanol (Bolton $\mathrm{et}$ al., 1965) and diluted to $2 \mathrm{M}$. The fatty acid standards were either from Applied Science Labs (State College, Pa., USA; hydroxy-fatty acids) or Supelco Inc. (Bellafonte, Pa., USA; n-fatty acids). Amino sugars (HCl-form) and 2-keto-3-deoxy-octonate (KDO; $\mathrm{NH}_{3}$-form) were from Sigma. The neutral sugars were either from BDH or Merck. The Fusobacterium nucleatum F1 LPS were isolated in our laboratory from whole cells by extraction with $45 \%(v / v)$ aqueous phenol.

Isolation and purification of LPS. The biomass from all strains was treated with pronase (Pronase B-grade, Calbiochem) (Chester \& Murray, 1975), and thereafter extracted with 45\% aqueous phenol under constant stirring for $30 \mathrm{~min}$ at room temperature (approximately $20^{\circ} \mathrm{C}$ ). The LPS was purified from the water phase by ultracentrifugation ( $100000 \mathrm{~g}$ for $90 \mathrm{~min}$ ) and treatment with ribonuclease as previously described (Nass \& Hofstad, 1982. 1984).

Degradation of LPS. Samples of freeze-dried LPS were hydrolysed with $1 \%$ acetic acid $(5 \mathrm{ml})$ at $100{ }^{\circ} \mathrm{C}$ for $90 \mathrm{~min}$ (Wilkinson et al., 1973). The hydrolysates were separated into chloroform-soluble, water-soluble and interfacial materials by thorough mixing with equal volumes of chloroform, followed by low-speed centrifugation. The chloroform layer was isolated, washed with water and concentrated to dryness under $\mathrm{N}_{2}$ to give lipid $\mathrm{A}$. The aqueous layer was washed with chloroform and dried in vacuo to give polysaccharide. The interfacial material was washed both with chloroform and with water, and dried in vacuo. The chloroform and water used for washing were added to their respective layers before concentration and drying.

Gel filtration. This was done through columns of Bio-Gel P-60 (100-200 mesh, exclusion limit 60000 daltons $)$ and Bio-Gel P-10 (100-200 mesh, exclusion limit 20000 daltons) (Bio-Rad). Pyridine/acetic acid buffer, pH 5.4 (10 ml pyridine and $4 \mathrm{ml}$ glacial acetic acid $\left.1^{-1}\right)$ was used as eluent. The fractionation was monitored by differential refractometry (RefractoMonitor 1107 , Laboratory Data Control, FI., USA). The void volume $\left(V_{0}\right)$ of the Bio-Gel P-60 column was $59.4 \mathrm{ml}$ (Blue dextran) and the total volume $\left(V_{t}\right)$ was $142.2 \mathrm{ml}\left(\mathrm{CuSO}_{4}\right)$. The corresponding figures for the Bio-Gel P-10 column were 14.2 and $44.0 \mathrm{ml}$.

Gas-liquid chromatography (GLC). Neutral sugars. Samples were hydrolysed in $0.1 \mathrm{M}-\mathrm{HCl}$ at $100{ }^{\circ} \mathrm{C}$ for $48 \mathrm{~h}$ in sealed glass tubes, neutralized with Amberlite IRA 410 ( $\mathrm{HCO}_{3}^{-}$-form), and the aldoses converted to alditol acetates (Sawardeker et al., 1965). GLC was done using a Perkin-Elmer $\mathbf{9 0 0}$ gas chromatograph with a flame ionization detector and a glass column $(0.2 \times 200 \mathrm{~cm})$ packed with $3 \%$ SP 2340 on Supelcoport 100-200 mesh (Supelco Inc.). $\mathbf{N}_{2}\left(30 \mathrm{ml} \mathrm{min}^{-1}\right)$ was used as carrier gas. The injector and detector temperatures were $250^{\circ} \mathrm{C}$, and the oven temperature was programmed from 180 to $250^{\circ} \mathrm{C}$ at a rate of $2^{\circ} \mathrm{C} \mathrm{min}-1$. D-Xylose was used as internal standard.

Amino sugars. Samples were hydrolysed overnight in $2 \mathrm{M}-\mathrm{HCl}$ at $100^{\circ} \mathrm{C}$. Acid was removed by evaporation in vacuo over $\mathrm{NaOH}$ pellets. The sugars were converted to their $O$-methyloxime acetates by the method of Mawhinney et al. (1980). GLC was done as described above, but the oven was programmed from 210 to $240^{\circ} \mathrm{C}$. The final temperature was held for $8 \mathrm{~min}$. Sorbitol was used as internal standard.

Fatty acids. Samples were methanolysed with $2 \mathrm{M}-\mathrm{HCl}$ in methanol at $85^{\circ} \mathrm{C}$ for $18 \mathrm{~h}$ (Jantzen et al., 1974). The methyl esters were extracted with hexane and treated with $50 \%(\mathrm{v} / \mathrm{v})$ trifluoroacetic anhydride in acetonitrile (TFAA) (Jantzen et al., 1978). GLC was done with the Perkin-Elmer gas chromatograph. The glass column (0.2 $\times 200 \mathrm{~cm}$ ) was packed with $3 \%$ SE-30 on Supelcoport $80-100$ mesh. The injector and detector temperatures were $200^{\circ} \mathrm{C}$, and the oven temperature was programmed from 120 to $240^{\circ} \mathrm{C}$ at a rate of $2^{\circ} \mathrm{C} \mathrm{min}-1, n$-Heptadecanoic acid was used as internal standard.

'Total analyses.' Methanolysed samples of material were also derivatized with TFAA as described by Bryn \& Jantzen (1982) for the simultaneous determination of monosaccharides and fatty acids. Methanolysates were dried under $\mathbf{N}_{2}$, re-evaporated with 1 vol. methanol and derivatized with $50 \%$ TFAA. After heating under reflux for $2 \mathrm{~min}$, the samples were dried and resuspended in 10\% TFAA. The samples were examined by GLC using a Hewlett-Packard 5710 gas chromatograph with a flame jonization detector and a glass capillary column (0.32 mm $\times 20 \mathrm{~m}$ ) coated at the Dept of Pharmacology, University of Bergen, with a $0.2 \mu \mathrm{m}$ film of SE-54 (bonded-phase: Crompack. Middelburg, Holland). He $\left(3 \mathrm{ml} \mathrm{min}^{-1}\right)$ was used as carrier gas. The injector and detector temperatures were $250^{\circ} \mathrm{C}$ and the oven temperature was programmed from 90 to $260^{\circ} \mathrm{C}$ at a rate of $8^{\circ} \mathrm{C} \mathrm{min}{ }^{-1}$. The initial temperature was held for $4 \mathrm{~min}$. $n$-Heptadecanoic acid was used as internal standard.

All gas chromatographic peak identities were established by comparing the relative retention time to those of authentic standards of neutral sugars, amino sugars (HCL-form), fatty acids, KDO and thenaolamine. In cases of 
doubt, the samples were spiked with derivatized authentic standards. To discriminate between the D-and L-forms of glycero-D-manno-heptose, LPS of Fusobacterium nucleatum F1, containing both these aldoheptoses (Fredriksen \& Hofstad, 1978), served as the standard. The respective response factors of the individual sugars and fatty acids were taken into account in quantification.

Colorimerric analyses. KDO was determined by a modification of the Weissbach \& Hurwitz method (Karkhanis et al., 1978). Samples were hydrolysed with $0.04 \mathrm{M} \cdot \mathrm{H}_{2} \mathrm{SO}_{4}$. Phosphorus was determined by a modification of the Fiske \& Subbarow method (Youngburg \& Youngburg, 1930). Protein was measured by the method of Bradford (1976), using the Bio-Rad Protein Assay.

Liberation of forty acids from lipid A. The lipid A was treated with alkaline hydroxylamine in ethanol for 3 min at $63^{\circ} \mathrm{C}$ (Snyder \& Stephens, 1959; Rietschel et al., 1972). The resulting suspension was centrifuged at $16000 \mathrm{~g}$ for I h. The supernatant, containing the formerly ester-linked fatty acids, was acidified with $2 \mathrm{M}-\mathrm{HCl}$ (approximately $\mathrm{pH}$ 1), heated for $1 \mathrm{~h}$ at $100^{\circ} \mathrm{C}$, and the liberated fatty acids were extracted with chloroform. The de-O-acylated lipid $\mathrm{A}$ present in the sediment was extracted with chloroform as well. In case any unextracted complete, or de- $O$ acylated lipid A remained, the sediment was treated with $4 \mathrm{M}-\mathrm{NaOH}$ at $100^{\circ} \mathrm{C}$ for $5 \mathrm{~h}$ to liberate the amide-linked fatty acids, and the acids were extracted with chloroform. The three chloroform extracts were dried under $N_{2}$. methanolysed, derivatized with TFAA and analysed by GLC.

\section{RESULTS}

With the exception of the LPS from strains E $8035(43.9 \mathrm{mg})$ and NCTC $11392(3.8 \mathrm{mg}), 20$ $30 \mathrm{mg}$ LPS was subjected to weak acid hydrolysis. The recovery of freeze-dried materials was $77-87 \%$. For most LPS approximately $35 \%$ of the recovered material was water-soluble (polysaccharide) and $40 \%$ chloroform-soluble (lipid A). The remaining material ended as an interface. The polysaccharide isolated from strain NCTC 11392 made up a smaller part of the hydrolysed LPS (12.9\%). This LPS also had the highest content of lipid A (54.8\%). The LPS of strain 11041 contained $53.4 \%$ polysaccharide, and that of strain $1110150.9 \%$ lipid A. Trifluoroacetic anhydride derivatives of LPS and of the materials isolated from the three layers of all six strains were analysed by GLC for sugar and fatty acid constituents. Typical patterns are shown in Fig. 1. The LPS and the polysaccharide of strain NCTC 11168 were contaminated with ribose due to incomplete ribonuclease treatment during isolation. The materials obtained in the various layers from strain E $\mathbf{8 0 3 5}$ were subjected to separate analyses for neutral sugars, amino sugars, fatty acids, KDO, phosphorus and protein. The analysis accounted for $44 \%$ of the polysaccharide, $53 \%$ of the lipid $\mathrm{A}$ and $29 \%$ of the interfacial material. The only additional information thus revealed was the presence of phosphorus in all three layers $(1.6 \%$ of the polysaccharide, $2.4 \%$ of lipid $A$ and $1.7 \%$ of the interfacial material) and that the protein present in the LPS remained with the lipid A.

With the exception of glucosamine, the majority of lipid As contained negligible amounts of sugars. An exception was the lipid A of strain E 8035, which contained $8.8 \%$ neutral sugars (determined as alditol acetates) in molar ratios similar to that of the parent LPS. The fatty acids present in the lipid As were $n$-tetradecanoic, 3-hydroxytetradecanoic and $n$-hexadecanoic acids either in molar ratios similar to those of undegraded LPS $(14: 0 / 3-\mathrm{OH}-14: 0 / 16: 0=0 \cdot 1 / 1 \cdot 0 / 0 \cdot 5-$ 1.3), or the amount of $16: 0$ was slightly higher. The supernatant after the hydroxylaminolysis contained the same acids, but the amounts of $14: 0$ and $16: 0$ were higher. The de- $O$-acylated lipid A contained 3-OH-14:0 only, or this acid in mixture with small amounts of $14: 0$ and $16: 0$. The $\mathrm{NaOH}$-treated sediment had either a fatty acid composition similar to untreated lipid $\mathrm{A}$ (strains NCTC 11168 and 11101 ), or there was an increase in the relative amount of 3-OH-14:0 (strains NCTC 11351 and 11041), or the latter was the only acid present (strains NCTC 11392 and $\mathrm{E}$ 8035).

The interfacial material had a qualitative chemical composition similar to unhydrolysed LPS, but a relatively higher content of fatty acids indicated that the material consisted of a mixture of LPS and lipid A. The material from strain 11101 had a composition identical to lipid A.

\section{Gel filtration of the polysaccharides}

The water-soluble polysaccharides contained neutral sugars, amino sugars, ethanolamine and KDO. With the exception of the material from strain E 8035, which contained $0 \cdot 3 \%$ of $16: 0$, the polysaccharides were free of fatty acids. All polysaccharides were chromatographed on Bio-Gel 

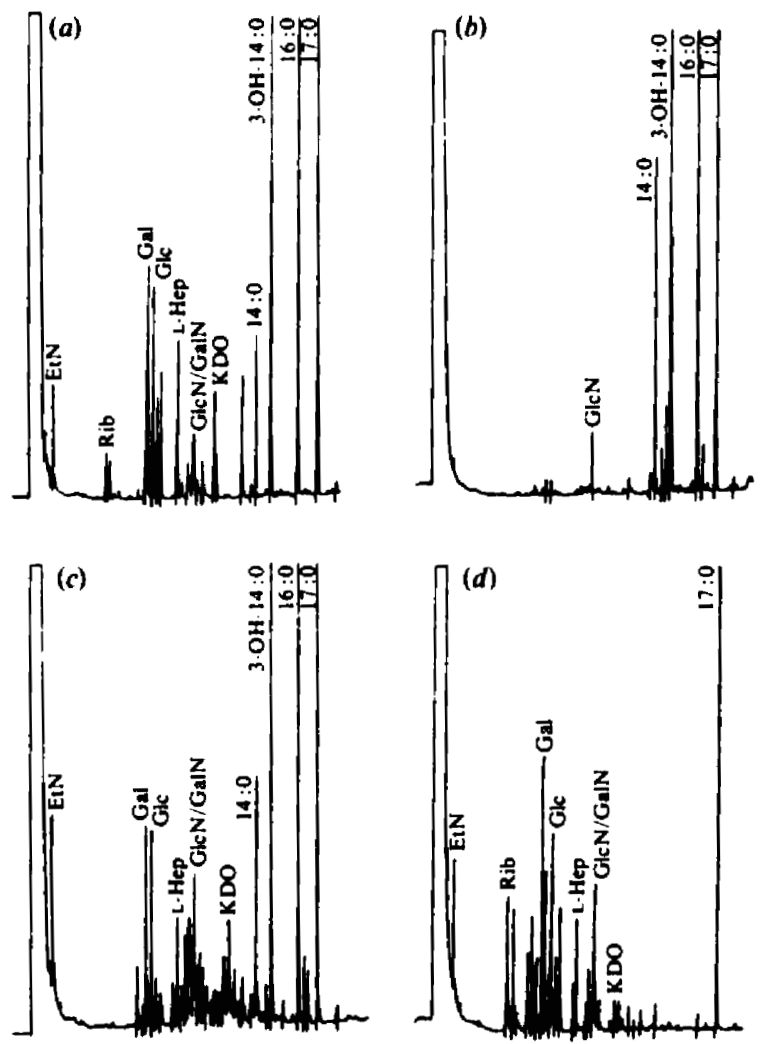

Fig. 1. Chromatograms of trifluoroacetic anhydride-derivatized LPS (a), lipid A (b), interfacial material (c) and polysaccharide (d) from strain NCTC 11168 . $n$-Heptadecanoic acid $(17: 0)$ was used as internal standard. The gas-liquid chromatographic data are reported in the text.

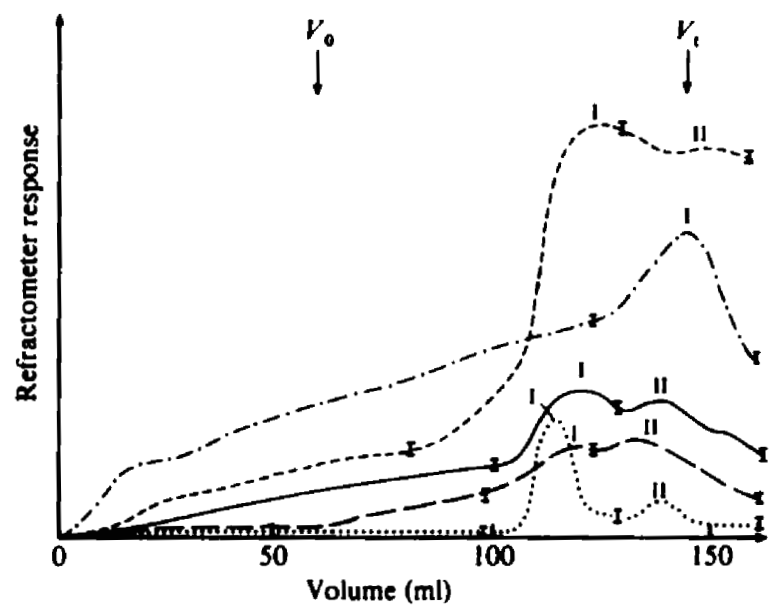

Fig. 2. Elution profile on Bio-Gel P-60 of degraded polysaccharide from LPS of $C$. jejuni NCTC 11168 (-). NCTC $11351(--)$ and $11041(--)$, and $C$. coli E $8035(-\cdot-)$ and $11101(\cdots \cdots \cdots \cdots)$ Fractions corresponding to I and II were collected as indicated by I- - I. $V_{0}$, void volume: $V_{1}$, total volume. 
Table 1. Molar ratios of sugars present in peak matrials obtained after gel filtration on Bio-Gel P-60 of water-soluble polysaccharide from degraded $L P S$ of $C$. jejuni and C. coli

\begin{tabular}{|c|c|c|c|c|c|c|c|c|c|}
\hline \multirow[b]{2}{*}{ Species } & \multirow[b]{2}{*}{ Strain } & \multirow[b]{2}{*}{ Material } & \multirow{2}{*}{$\begin{array}{c}\text { Peak } \\
\text { maximum } \\
\text { with P-60 } \\
\text { column }\end{array}$} & \multicolumn{5}{|c|}{ Molar ratios oft: } & \multirow{2}{*}{$\begin{array}{l}\text { Peak } \\
\text { maximum } \\
\text { with P-10 } \\
\text { column } \downarrow\end{array}$} \\
\hline & & & & Gal & Glc & L-Hep & AS & KDO & \\
\hline C. jejuni & NCTC 11351 & $\begin{array}{l}\text { LPS } \\
\text { Polysaccharide } \\
\text { Peak I } \\
\text { Peak II } \\
\text { LPS } \\
\text { Polysaccharide } \\
\text { Peak I } \\
\text { Peak II } \\
\text { LPS } \\
\text { Polysaccharide } \\
\text { Peak I } \\
\text { Peak II }\end{array}$ & $\begin{array}{l}- \\
- \\
2 \cdot 0 \\
2 \cdot 3 \\
- \\
- \\
2 \cdot 0 \\
2 \cdot 2 \\
- \\
- \\
2 \cdot 1 \\
2 \cdot 5\end{array}$ & $\begin{array}{l}3 \cdot 2 \\
3 \cdot 4 \\
3 \cdot 1 \\
2 \cdot 4 \\
1 \cdot 3 \\
1.9 \\
1.9 \\
1 \cdot 3 \\
2 \cdot 6 \\
1.0 \\
2.5 \\
3 \cdot 4\end{array}$ & $\begin{array}{l}2 \cdot 8 \\
3 \cdot 6 \\
3 \cdot 6 \\
5 \cdot 0 \\
2 \cdot 4 \\
3 \cdot 3 \\
3 \cdot 3 \\
2.8 \\
2 \cdot 2 \\
1 \cdot 7 \\
2.9 \\
5 \cdot 2\end{array}$ & $\begin{array}{l}1.0 \\
1.0 \\
1.0 \\
1.0 \\
1.0 \\
1.0 \\
1.0 \\
1.0 \\
1.0 \\
1.0 \\
1.0 \\
1.0\end{array}$ & $\begin{array}{l}1.6 \\
2.1 \\
1.6 \\
3.1 \\
1.2 \\
1.8 \\
1.5 \\
1 \cdot 3 \\
0.4 \\
1.1 \\
1.0 \\
3.4\end{array}$ & $\begin{array}{l}1 \cdot 2 \\
0 \cdot 6 \\
0 \cdot 3 \\
4 \cdot 9 \\
1 \cdot 1 \\
1 \cdot 1 \\
0 \cdot 2 \\
2 \cdot 1 \\
0 \cdot 3 \\
0 \cdot 6 \\
0 \cdot 4 \\
2 \cdot 6\end{array}$ & $\begin{array}{l}- \\
\overline{2 \cdot 8} \\
2.9 \\
- \\
\overline{2.9} \\
3.0 \\
- \\
- \\
2.8 \\
2.9\end{array}$ \\
\hline C. coli & 11101 & $\begin{array}{l}\text { LPS } \\
\text { Polysaccharide } \\
\text { Peak I } \\
\text { LPS } \\
\text { Polysaccharide } \\
\text { Peak I } \\
\text { Peak II }\end{array}$ & $\begin{array}{c}- \\
\bar{C} \\
2 \cdot 4 \\
- \\
1.9 \\
2 \cdot 3\end{array}$ & $\begin{array}{l}0.7 \\
0.4 \\
0.7 \\
0.5 \\
1.3 \\
0.9 \\
0.7\end{array}$ & $\begin{array}{l}1.2 \\
0.7 \\
1.1 \\
1.0 \\
1.4 \\
1.5 \\
1.5\end{array}$ & $\begin{array}{l}1.0 \\
1.0 \\
1.0 \\
1.0 \\
1.0 \\
1.0 \\
1.0\end{array}$ & $\begin{array}{l}1 \cdot 1 \\
0.7 \\
0.8 \\
0.8 \\
1.3 \\
0.7 \\
3 \cdot 1\end{array}$ & $\begin{array}{l}0.8 \\
0.4 \\
0.6 \\
0.6 \\
0.8 \\
0.2 \\
3 \cdot 8\end{array}$ & $\begin{array}{l}- \\
- \\
2 \cdot 7 \\
- \\
2 \cdot 2 \\
3 \cdot 1\end{array}$ \\
\hline
\end{tabular}

- Elution volume $\times$ void volume.

$\uparrow$ GalN and GlcN in C. jejuni, GlcN in C. coli: L-Hep, L-glycerol-D-manno-heptose; AS, amino sugars; KDO, 2-keto-3-deoxy-octonate.

$\ddagger$ Elution volume $\times$ void volume, obtained by re-chromatography of the Bio-Gel P-60 peak materials.

P-60 (Fig. 2). Because of the small amount available, the polysaccharide of LPS from strain NCTC 11392 was chromatographed on a much smaller P-60 column $\left(v_{0}=11.0 \mathrm{ml}, V_{1}=\right.$ $16.5 \mathrm{ml}$, i.e. $1.5 \times V_{0}$ ) (elution profile not shown). The polysaccharides from the LPS of strains E 8035 and NCTC 11392 formed one peak, having its maximum at the total volume of the columns. The polysaccharides from the LPS of the other strains showed one peak (I) just before, and another peak (II) at the total volume of the column. The peak I of the polysaccharide from strain 11101 had a maximum closer to the void volume than the polysaccharide from the other LPS (Table 1).

For all strains, except 11392, samples of the fractions eluted before the peaks, and of the peakforming materials, were derivatized with TFAA for GLC. The eluate before peak I contained either small amounts of glucose or amino sugars or both. Peaks I and II contained the same components as the degraded polysaccharide. The main difference between the two peaks was a much higher content of amino sugars and KDO in peak II. For most strains the relative amounts of neutral sugars resembled those of the parent polysaccharides (Table 1). Hydrolysed and unhydrolysed samples of the peak-forming material from the polysaccharide of LPS from strain E 8035 were subjected to separate analysis for neutral sugars: $32.2 \%$ of the material was identified as neutral sugars after hydrolysis, as compared to $2.3 \%$ without hydrolysis. The relative amounts of glucose and galactose were higher in the unhydrolysed, as compared to the hydrolysed, samples.

The peak-forming materials from all strains, except NCTC 11392, were re-chromatographed using a Bio-Gel P-10 column. The peak II materials formed a peak having its maximum a little closer to the total volume than the material of peak 1 . A shoulder formed on the peak I material of strain NCTC 11168 which indicated that this material was heterogenous in composition. The peak I material from strain 11101 was eluted closer to the void volume of the column than the peak I material from the other polysaccharides. A sample of water-soluble polysaccharide from degraded LPS from Escherichia coli Su 4411-41 (O14:K7:H-, core type R4) showed an elution 
profile similar to the peak-forming material from strain E 8035 when chromatographed on the same P-10 column. Samples of the peak-forming materials obtained following the P-10 column were derivatized with TFAA for GLC. The gas chromatograms did not reveal any further information, except that for some polysaccharides the high amount of KDO present in the peak II material was reduced.

\section{DISCUSSION}

The LPS from $C$. jejuni and $C$. coli were readily split by treatment with $1 \%$ acetic acid into chloroform-soluble lipid $\mathrm{A}$ and water-soluble, KDO-containing polysaccharide. This indicates that the lipid A and the polysaccharide part of the Campylobacter LPS are linked via KDO, as is the case with enterobacterial LPS.

The high content of fatty acids relative to neutral sugars in the interface indicates that lipid $A$ constitutes a greater part of the LPS macromolecule than that indicated by the percentage amount of lipid $A$ isolated. Hydroxylaminolysis shows that 3-OH-14:0 is present in lipid A as ester- and amide-linked fatty acid residues, while $16: 0$ and 14:0 are ester-linked. The Campylobacter lipid As contain both glucosamine and phosphate. These two compounds are found in the enterobacterial lipid $A$ where the $\beta$-1,6-linked D-glucosamine residues substituted by ester- and glycosidically-linked phosphate groups form a backbone. The lipid $A$ backbone is one of the more conservative structural forms found among the Gram-negative bacteria. A similar structure may be present in Campylobacter LPS. The low yield of glucosamine (cf. Fig. $1 b$ ) can be explained by the fact that phosphorylated sugars are undetectable by GLC. In addition, only glycosidically linked phosphate groups are released by the hydrolytic conditions used. The results of the experiments carried out on the chloroform-soluble material indicate that the main structure of the $C$. jejuni and $C$. coli lipid As resembles that of enterobacterial lipid A.

The typical elution profile from gel filtration on Bio-Gel P-60 columns of the water-soluble polysaccharide obtained by weak acid hydrolysis of enterobacterial S-form LPS shows a peak appearing at the void volume of the column and another peak near the total volume. The first peak corresponds to polymeric O-antigenic side-chains, with or without attached core fragments, and the second to the core oligosaccharide. The water-soluble polysaccharide of the LPS of $C$. coli E 8035 and $C$. jejuni NCTC 11392 was eluted from the Bio-Gel P-60 columns as a homogeneous peak having a maximum at the total volume of the column, indicating that both LPS are devoid of polymeric side-chains. The elution profile from the gel filtration on the BioGel P-10 column of the LPS from strain E 8035 is similar to that of the polysaccharide of the LPS from $E$. coli Su 4411, which is a core oligosaccharide made up of 10 sugar residues (Glc: Gal : LHep in ratios 3:4:3) (Ørskov et al., 1977). This suggests that the former is an R-type oligosaccharide composed of KDO, L-glycero-D-manno-heptose, glucose, galactose and glucosamine. A prerequisite for this assumption is that the two oligosaccharides are similar in shape. One or more of the sugar residues and/or the ethanolamine is phosphorylated. The result of GLC of the unhydrolysed samples indicates that galactose and glucose are in a terminal position. Other bacterial species having ' $R$ '-type LPS as their naturally occurring $O$-antigens also exist. Jennings et al. (1979) found LPS of all the different serogroups of Neisseria meningitidis to be ' $R$ '-types by gel filtration of the degraded polysaccharide. Tsai et al. (1983) have verified these results using SDS-PAGE.

Gel filtration of the four other water-soluble polysaccharides indicates heterogeneity with respect to the molecular weight of the polysaccharide portion of the LPS, or that some fragmentation had taken place during the degradation with acetic acid. Heterogeneity with respect to $\mathrm{O}$-antigenic side-chain length is a common phenomenon in phenol/water extracted LPS (Jann et al., 1975; Goldman \& Leive, 1980; Palva \& Mäkelä, 1980). The elution profiles from the Bio-Gel P-60 gel filtration, and the GLC findings of approximately 10 to 25 hexose residues per 3 residues of heptose, may indicate that short 0 -antigenic side-chains are present in these LPS. The results from the gel filtration of the different water-soluble polysaccharides are comparable to those reported by Logan \& Trust (1982). They found their $C$. coli and $C$. jejuni LPS preparations to have an electrophoretic mobility on SDS-PAGE suggestive of LPS composed of lipid A and core oligosaccharide. This was in contrast to LPS isolated from other Campylobacter species. 
We are grateful to E. Solheim. Dept. of Pharmacology, University of Bergen, Norway, for his aid in the production of the capillary columns.

\section{REFERENCES}

Bolton, C. M., Clamp, J. R. \& Hough, L. (1965). The use of gas-liquid chromatography in investigations on glycoproteins. Biochemical Journal 96, Sc.

Bradford, M. M. (1976). A rapid and sensitive method for the quantification of microgram quantities of protein utilizing the principle of protein-dye binding. Analytical Biochemistry 72, 248-254.

Bryn, K. \& JANTZEN, E. (1982). Analysis of lipopolysaccharides by methanolysis, trifluoroacetylation, and gas chromatography on a fused-silica capillary column. Journal of Chromatography 240, 405-413.

Chester, I. R. \& Murray, R. G. E. (1975). Analysis of the cell-wall and lipopolysaccharide of Spirillum serpens. Journal of Bacteriology 124, 1168-1176.

Frederiksen, G. \& Hofstad, T. (1978). Chemotypes of Fusobacterium nucleatum lipopolysaccharides. Acta pathologica et microbiologica scandinatica, Section B, 86, 41-45.

George, H. A., hoffman, P. S., Krieg, N. R. \& SMIBERT, R. M. (1978). Development of an improved culture medium for Campylobacter ferus. Journal of Clinical Microbiology 8, 36-41.

Golduan, R. C. \& Leive, L. (1980). Heterogeneity of antigenic sidechain length in lipopolysaccharides from Escherichia coli OIII and Salmonella typhimurium LT2. European Journal of Biochemistry 107, 145153.

JANN, B., RESKE, K. \& JANN, K. (1975). Heterogeneity of lipopolysaccharides. Analysis of polysaccharide chain-lengths by sodium dodecylsulfate-polyacrylamide gel electrophoresis. European Journal of Biochemistry, 60, 239-246.

Jantzen, E., Bryn, K. \& Bovre, K. (1974). Gas chromatography of bacterial whole cell methanolysates. IV : A procedure for fractionation and identification of fatty acids and monosaccharides of cellular structures. Acta pathologica et microbiologica scandinarica, Section B, 82, 753-766.

Jantzen, E., Bryn, K., Hagen, N.. Bergan, T. \& Bovre, K. (1978). Fatty acids and monosaccharides of Neisseriaceae in relation to established taxonomy. NIPH Annals (Oslo) 1, 59-71.

Jennings, H. J., Battacherjee, A. K., Kenne, L., Kenny, C. P. \& Calver, G. (1979). The R-type lipopolysaccharides of Neisseria meningitidis. Canadian Journal of Biochemistry and Physiology 58, 128-136.

KarkHanis, Y., Zeltner, J. Y., JaCKson, J. J. \& Carlo, D. J. (1978). A new and improved microassay to determine 2-keto-3-deoxy-octonate in lipopolysaccharide of gram-negative bacteria. Analytical Biochemistry 85, 595-601.
Logan, S. \& Trust, T. J. (1982). Outer membrane characteristics of Campylobacter jejuni. Infection and Immunity 38, 898-906.

Mawhinney, J. R., Feater, M. S., Barbero, G. J. \& MARtinez, J. R. (1980). The rapid, quantitative determination of neutral sugars (as aldonitrile acetates) and amino sugars (as $\boldsymbol{O}$-methyloxime acetates) in glycoproteins by gas-liquid chromatography. Analytical Biochemistry 110, 112-117.

Ness, V. \& HofsTAD, T. (1982). Isolation and chemical composition of lipopolysaccharides from Campylobacter jejuni. Acta pathologica et microbiologica scandinarica, Section B, 90, 135-139.

N ess, V. \& Hofstad, T. (1984). Chemical composition and biological activity of lipopolysaccharides prepared from type strains of Campylobacter jejuni and Campylobacter coli. Acta pathologica et microbiologica' scandinatica, Section B (in the Press).

Grskov, I., Brskov, F., JANN, B. \& JANN, K. (1977). Serology, chemistry and genetics of $O$ and $R$ antigens of Escherichia coli. Bacteriological Review's 41, 667-710.

Palva, E. T. \& Mäkelä, P. H. (1980). Lipopolysaccharide heterogeneity in Salmonella typhimurium. Analysed by sodium dodecylsulfate/polyacrylamide gel electrophoresis. Eurpean Journal of Biochemistry 107, 137-143.

Rietschel, E. T., Gottert, H., Lüderitz, O. \& WestPhaL, O. (1972). Nature and linkages of the fatty acids present in the lipid A component of Salmonella lipopolysaccharides. European Journal of Biochemistry 28, 166-173.

Sawardeker, J. S., Sloneker, J. H. \& Jeans, A. (1965). Quantitative determination of monosaccharides as their alditol acetates by gas-liquid chromatography. Analytical Chemistry 37, 16021604.

SNYDER, F. \& StePhens, N. (1959). A simple spectrophotometric determination of ester groups in lipids. Biochimica et biophysica acta 34, 244-245.

Tsai, C.-M., Boykins, R. \& Frasch, C. E. (1983). Heterogeneity and variation among Neisseria meningitidis lipopolysaccharides. Journal of Bacteriology 155, $498-504$.

Wilkinson, S. G., Galbraith, L. \& Lightfoot, G. A. (1973). Cell-walls, lipids and lipopolysaccharides of Pseudomonas species. European Journal of Biochemistry 33, $158-174$.

YoungburG, G. E. \& Youngburg, M. V. (1930). Phosphorus metabolism. I. A system of blood phosphorus analysis. Journal of Laboratory and Clinical Medicine 16, 158-174. 